Artículo

\title{
VIOLENCIA HACIA LA MUJER EN LA ETAPA DE ENAMORAMIENTO A TRAVÉS DE LAS REDES SOCIALES
}

\author{
VIOLENCE TOWARDS WOMEN IN THE STAGE OF \\ FALLING IN LOVE THROUGH SOCIAL NETWORKS
}

\author{
July Marli Díaz Fernández (1) \\ Katia Anaís Pacheco Rodriguez (2) \\ Rosa Jeuna Díaz Manchay (3) \\ Mirtha Flor Cervera Vallejos (4) \\ Antonieta de Jesús Banda Pérez (5) \\ Lisseth Rodríguez Cruz (6)
}

1. Licenciada en enfermería. Escuela de Enfermería de la Universidad Católica Santo

Toribio de Mogrovejo Chiclayo, Perú.

2. Licenciada en enfermería. Escuela de Enfermería de la Universidad Católica Santo Toribio de Mogrovejo Chiclayo, Perú.

3. Doctora en Ciencias de Enfermería. Coordinadora de asuntos académicos de la Escuela de Enfermería de la Universidad Católica "Santo Toribio de Mogrovejo"-Chiclayo.

4. Doctora en Enfermería. Directora de Escuela de Postgrado de la Universidad Católica Santo Toribio de Mogrovejo Chiclayo, Perú.

5. Estudiante de Doctorado en Ciencias de Enfermería, Campus Celaya Salvatierra Universidad de Guanajuato. Docente de la Facultad de Enfermería y Nutrición de la Universidad Autónoma de San Luis Potosí.

6. Doctoranda en Ciencias de Enfermería de la Universidad de Navarra-España. Docente de la Escuela de Enfermería de la Universidad Católica Santo Toribio de Mogrovejo Chiclayo, Perú. 
Correspondencia: Rosa Jeuna Díaz Manchay, correo: rdiaz@usat.edu.pe

Recibido el: 06 de julio de 2018

Aceptado el: 24 de julio de 2018

\title{
Resumen
}

Objetivos: Describir la violencia a la mujer en la etapa de enamoramiento a través de las redes sociales según percepción de docentes y estudiantes de enfermería de una universidad privada. Métodos: Investigación cualitativa descriptiva, muestra fue de 20 informantes. Resultados: Control, manipulación y denigración a través de las redes sociales. Conclusión: El uso de celulares y las redes sociales como el Facebook, WhatsApp, Instagram, entre otros, se han convertido en fuente de vigilancia y control hacia la mujer, el enamorado como muestra de amor conoce la "clave", y hace seguimiento de las publicaciones, conversaciones y amistades; convirtiéndose en un medio de manipulación y denigración, pues la mujer es dominada y amenazada con exhibir fotos o videos privados.

Palabras clave: Docentes, estudiantes, violencia contra la mujer, redes sociales.

\begin{abstract}
Objectives: To describe the violence to women in the stage of falling in love through social networks according to the perception of teachers and nursing students of a private university. Methods: Descriptive qualitative research, sample was 20 informants. Results: Control, manipulation and denigration through social networks. Conclusion: The use of cell phones and social networks such as Facebook, WhatsApp, Instagram, among others, have become a source of surveillance and control towards women, the lover as a sign of love knows the "key", and follows up on the publications, conversations and friendships; becoming a means of manipulation and denigration, since women are dominated and threatened with exhibiting private photos or videos.
\end{abstract}


Key Words: Teachers, students, violence against women, social networks

\section{INTRODUCCIÓN}

La violencia hacia la mujer se considera un problema de salud pública; es una violación de los derechos humanos, un acto en el que la mujer puede estar sujeta a sufrir daños físicos, sexuales y psicológicos, que disminuyen su calidad de vida y su propia valía como ser humano ${ }^{(1)}$. A pesar de las leyes promulgadas, la prevalencia de la violencia en la pareja sigue siendo uno de los problemas más preocupantes en el siglo XXI, y llega a comportar considerables costes y consecuencias graves para las víctimas ${ }^{(2)}$. Además, la violencia en la etapa del enamoramiento suele permanecer en una situación oculta, en la que el agresor gradualmente manifiesta su conducta de control y violencia para imponer su voluntad, conductas que limitan actuaciones de la mujer y la convierten en un ser silencioso, abatida y deprimida ${ }^{(3)}$.

Actualmente, las redes sociales se usan para intimidar, controlar a la pareja, usurpar la personalidad e incluso como violación de la intimidad tras las rupturas de esta. Entre los motivos que aluden las parejas que ya han experimentado estas rupturas, enfrentamientos o incluso episodios violentos de celos, son fundamentalmente por conexiones a deshora que después no se pueden explicar y por las conductas obsesivas de estar en permanente contacto, propiciadas por el uso del WhatsApp. Una respuesta a destiempo, cuando se sabe que la otra persona ha recibido el mensaje, puede ocasionar, en estos casos, conflicto. Y también conectarse a una hora en la que el otro miembro de la pareja cree que la persona querida está haciendo otra cosa ${ }^{(4)}$. Situación corroborada por el estudio de Estébanez ${ }^{(5)}$ quien concluye que la violencia se da cuando el enamorado ejerce un control y vigilancia constante de todas las cosas que la enamorada hace. Pero, las relaciones actuales están inmersas en las redes sociales, lo que puede tener ventajas, y a la vez pueden ser fuente de conflicto, pues permite la supervisión constante de la pareja, generando inquietudes y 
desconfianza ${ }^{(6)}$. Además la creencia que los celos son una prueba de amor, coexiste como uno de los resultados más preocupantes, ya que esta confusión puede derivar en el primer estadio del maltrato, sin que se sea consciente de ello ${ }^{(7)}$.

Hoy en día, la violencia en el enamoramiento se da en cualquier cultura o estatus social, tanto así que se puede evidenciar en colegios, zonas rurales y hasta en la misma ciudad. Muchas veces, por vergüenza o el temor de represalias, las mujeres víctimas de violencia callan y no denuncian estos hechos a las instancias responsables. Al respecto, las investigadoras han observado en su vida familiar y en la universidad, que algunas estudiantes son acosadas por sus enamorados, ellos saben exactamente sus horarios, no pueden salir solas a reuniones, a veces las esperan fuera de su salón de clase, y cuando tardan en salir o no contestan el celular, las gritan o jalonean del brazo, las obligan a colocar en su foto de perfil del Facebook a su pareja para que nadie las mire, o saben sus claves de correo y de sus cuentas de redes sociales. Los objetivos fueron: Describir la violencia a la mujer en la etapa de enamoramiento a través de las redes sociales según percepción de docentes y estudiantes de la escuela de enfermería de una Universidad Privada en Lambayeque, Perú.

\section{MÉTODO}

La presente investigación fue de tipo cualitativa, con abordaje descriptivo ${ }^{(8)}$. Los sujetos de estudio lo constituyeron 10 docentes que fueron coordinadoras de asignaturas y 10 estudiantes de Enfermería del X ciclo de una universidad privada en Lambayeque-Perú, quienes develaron la percepción acerca de la violencia a la mujer en la etapa de enamoramiento a través de las redes sociales. La confidencialidad, tanto para docentes y estudiantes fueron aseguradas por códigos de identificación. Para las estudiantes se usó UME1... UME10 y para las docentes EDE1...EDE10. El muestreo fue por conveniencia, y la muestra determinada por la técnica de saturación y redundancia. Para la recolección de datos se utilizó la entrevista semiestructurada ${ }^{(9)}$, la cual fue elaborada por las investigadoras, validada por juicio de expertos, y prueba piloto. Después de la aprobación 
del Comité de Ética de Investigación de la Facultad de Medicina de la Universidad Católica Santo Toribio de Mogrovejo, se procedió a ubicar a los sujetos de estudio y según su disponibilidad se realizaron las entrevistas previa firma del consentimiento informado. Cada entrevista duró alrededor de 30 minutos, mediante un grabador de voz, papel y lápiz, el cual tuvo como fin captar los datos brindados en su totalidad.

La información obtenida se procesó por análisis de contenido temático, técnica de interpretación de textos. Tuvo tres etapas (9), a continuación, descritas:

a) Pre análisis: Donde se seleccionó los documentos o información sometida para el análisis.

b) Codificación: Proceso a través del cual se fragmentaron los datos en función de su significación y posterior a una lectura en profundidad, se determinó la codificación en sí, mediante la identificación de palabras, frases o párrafos con significación destacable en relación a los objetivos de este estudio.

c) La categorización: Consistió en la segmentación de elementos singulares, o unidades, que resultaron relevantes y significativas desde el interés investigativo. Se agruparon las unidades seleccionadas por similitud en cuanto a su significado, estableciendo conexiones entre las mismas contrastando la realidad con artículos científicos.

En la presente investigación prevalecieron los principios de la ética personalista. Los sujetos de estudio no fueron sometidos a situaciones que les ocasione algún tipo de sufrimiento emocional, social y físico. Su identidad fue protegida por códigos y tuvieron la decisión de elegir si deseaban o no participar de la investigación. Así mismo la investigación se ha diseñado con rigor científico considerando básicamente tres criterios (8). La investigación estuvo validada mediante datos reales y verdaderos, teniendo en cuenta una revisión minuciosa de las transcripciones de las entrevistas de los sujetos del estudio, para ello las investigadoras retornaron al escenario de la investigación para confirmar los hallazgos y revisar algunos datos particulares. Los informantes colaboraron en corregir los errores de interpretación o trascripción de las entrevistas, para tener claro dicha información. Cabe mencionar el uso de un registro y documentación completa de 
las decisiones o ideas que las investigadoras tuvieron en el estudio, con la finalidad de que sirvan como base de datos para otras investigaciones relacionadas con el tema de investigación o como fuente de verificación de la misma.

\section{RESULTADOS}

\section{A. Control a la mujer en el enamoramiento a través de las redes sociales.}

Las redes sociales se han convertido en símbolo de control hacia la pareja, situación que se constata en los siguientes discursos de docentes y estudiantes:

“inconscientemente las redes sociales como el Facebook, WhatsApp, Instagram llegan hacer un vínculo de control y violencia, donde el hombre quiere tener el control absoluto de las cuentas privadas de su pareja, para vigilar con quien conversa, que fotos sube y así controlar su círculo de amigos” EDE7

"se da a través de las cuentas de las redes sociales (Facebook, WhatsApp, etc.) como una forma más rápida de acceder jaqueando o pidiendo las claves para mantenerla controlada...algunos exigen ponerlos en la foto de portado o de perfil, para que todos vean que somos pareja” EDE2, EDE9

“A veces los victimarios no controlan sus emociones y lastiman a su enamorada ya sea con atosigamiento y control donde la víctima no tiene libertad, ni tranquilidad... y esto se debe a la desconfianza, los celos, la baja autoestima, falta de control emocional ...el enamorado pide la contraseña y las chicas les dan porque es como una prueba de amor y confianza, así lo hacen sentir los chicos, aunque ellos no dan su clave" UME2, UME4 
"La violencia a la mujer pretende el control y sumisión, con ella quieren llegar a convertir a la mujer en una cosa de su propiedad para así poder hacer y deshacer sin consideración alguna hacia ella, hasta llegar a anularla completamente y lograr el total de dominio” UME7

Las docentes y estudiantes de enfermería declaran que el enamorado pide la contraseña de las redes sociales, por los celos, miedo e inseguridades que se generan a perder a la persona que "aman"; sin embargo, esto puede conllevar a asfixiar la relación.

\section{B. Manipulación y denigración a la mujer en el enamoramiento a través de las redes sociales.}

Docentes y estudiantes manifiestan que a través de las redes sociales se da la manipulación y denigración a la mujer en la etapa de enamoramiento.

"Las redes sociales se han vuelto un desencadenante para la violencia en la etapa de enamoramiento, ya que a través de ellas pueden manipular, denigrar, chantajear, humillar a su víctima a través de fotos, conversaciones y videos privados y llegar a un control máximo sobre ella” EDE5, EDE7, EDE10

\footnotetext{
“A veces en las parejas hay fotos o videos íntimos que se dan durante la relación, y cuando la mujer quiere poner fin a la relación el hombre utiliza estos medios tratando de amedrentarla o chantajearla diciendo que va a subir estas fotos o videos al Facebook, o mostrarlos por Whatsapp si es que lo deja, tratando de denigrar a la mujer con esos chantajes de no seguir con la relación”. UME1, UME5, UME8, UME10
}

Las redes sociales son un desencadenante para la violencia dado a través de la manipulación por fotos, conversaciones, videos íntimos, donde el victimario buscará 
manipular y chantajear a la mujer para acceder a aquello que él quiere, y si se niega a ello tendrá como consecuencia la difamación de su persona exponiéndola a la humillación pública, llegando a intimidarla y aislarla.

\section{DISCUSIÓN}

Los hallazgos de esta investigación reflejan que actualmente se ha incrementado el uso de la tecnología digital como los celulares. El 28,2\% de la población que usa internet lo hace exclusivamente por teléfono móvil y $88,8 \%$ de la población que navegó en Internet lo utilizó para comunicarse utilizando redes sociales como el Facebook, WhatsApp e Instagram ${ }^{(10)}$; sin embargo, dichas tecnologías se han convertido en fuente de control en la fase de enamoramiento y de expresión de violencia sobre todo a la mujer. Como refieren las informantes del estudio: "El hombre quiere tener el control absoluto de las cuentas privadas de su pareja, para vigilar con quien conversa, que fotos sube y así controlar su círculo de amigos". Según un estudio, los recursos en los que se percibe un mayor riesgo de sufrir violencia son facebook, whatsapp, ask.fm e instagram, recursos que permiten una interactividad, en cambio youtube destaca por ser percibido con menor riesgo ${ }^{(11)}$.

También, en las conclusiones de un estudio descubrieron que la violencia psicológica tiene una mayor presencia a través de las redes sociales; aunque no la sufre la mayoría de adolescentes, sí que es importante destacar que entre los y las adolescentes que participaron en este estudio, 2 de cada 10 chicas y 3 de cada 10 chicos declararon haber recibido algún tipo de amenaza a través de las redes sociales; 3 de cada 10 adolescentes habían sufrido algún tipo de insulto, malos comentarios o incluso humillaciones a través de las redes sociales o de un chat; cuando en las entrevistas en profundidad se les pregunto abiertamente si pensaban que las redes sociales eran un instrumento para ejercer el maltrato psicológico, todos contestaron abiertamente que sí ${ }^{(4)}$.

Según Donoso(11), colgar en Internet una imagen comprometida de la pareja o ex pareja; 
dar de alta, con foto incluida, a la víctima en una web donde se trata de votar a la persona de forma negativa; hacerte con los datos personales de tu pareja o ex pareja y publicarlos sin su permiso; crear un perfil o espacio falso en nombre de la víctima y decidir el contenido de sus publicaciones; y grabarse teniendo relaciones sexuales y colgarlo en la red, destacan porque no ocurren, tanto en la forma activa como pasiva, situándose los porcentajes de «nunca» en las respuestas, en torno al 98\%. En este estudio manifiestan: “A veces en las parejas hay fotos o videos íntimos que se dan durante la relación, y cuando la mujer quiere poner fin a la relación el hombre utiliza estos medios tratando de amedrentarla o chantajearla". Es alarmante como estos jóvenes usan la tecnología y redes sociales como parte de su vida cotidiana para todo tipo de comunicación ya sea asertiva o no.

Es así que el efecto que tienen las nuevas tecnologías en las relaciones de enamorados es la práctica de compartir la contraseña o negarse a ello puede traer consecuencias como la violencia verbal o física. Como manifiestan en el estudio: "El enamorado pide la contraseña y las chicas les dan porque es como una prueba de amor y confianza, así lo hacen sentir los chicos". Otorgar la contraseña de sus redes sociales o de su celular al enamorado, constituye una nueva prueba de amor que algunos jóvenes se exigen entre sí como muestra de confianza absoluta, prueba que unos rechazan y otros aceptan. Los principales conflictos que emergen en los enamorados asociados a los usos de tecnologías son por celos. En efecto los celos son unos de los más relevantes problemas que genera el uso de la tecnología, ya que permite controlar las actividades que realiza la otra persona, igual el hecho de subir fotografías y observar que a varias personas les guste la foto de su pareja, puede desatar una serie de problemas ${ }^{(11)}$. Los celos se convierten en una obsesión tienen lugar cuando comienzan las conductas policíacas de control: revisar el Facebook, entrar en el email, espiar el móvil, hacer un interrogatorio a la enamorada cada vez que sale de casa.

Según los estudios de investigación ${ }^{(5)(12)}$ y constatando con las entrevista de los sujetos de estudio las redes sociales se han vuelto un desencadenante, para la violencia a la mujer en la etapa de enamoramiento; queriendo llegar a la búsqueda de poder, control y dominio, 
denigración, aislamiento del enamorado a través de las redes sociales, donde hay evidencias de conductas violentas cuyo propósito es conseguir la dependencia total; obligando a su enamorada darle la contraseña o poniendo como prueba de amor entregar la contraseña para hacer un seguimiento continuo de sus conversaciones y así tener el control absoluto, pero muchas veces la mujer ve esto normal y justifica. Aludiendo que lo hace porque la quiere, y todo esto se debe ante la inseguridad, los celos y la desconfianza del agresor.

Por otra parte se encontró que las principales conductas relacionadas con la violencia de pareja son: intercambiar las contraseñas de las redes sociales, colgar en internet una imagen comprometida o datos que puedan perjudicar a la pareja o ex pareja, usurpar la clave de correo electrónico, amenazar con revelación de datos, vídeos o fotografías, y controlar las amistades de la pareja en las redes sociales así como las publicaciones que realiza. Dichas acciones reflejan el control que se quiere tener de la persona y como las redes sociales nos adentran más a estas situaciones vividas cada día y como estos medio tecnológicos es un factor de violencia de género.

También las estudiantes expresan como la manipulación y el control, del enamorado le crea la convicción que la enamorada está a su disposición y tiende a tener una vigilancia constante sobre su víctima privándola tanto de su vida social como de su libertad, llegando a ejercer en ella un chantaje psicológico o emocional provocando así un sentimiento de culpa, temor y sumisión en ella y que finalmente terminan cediendo a su manipulación.

\section{CONCLUSIONES}

La violencia a la mujer en la etapa de enamoramiento es percibida como manipulación, control y denigración por parte del agresor hacia su víctima. Y esto se da básicamente a través del uso de las redes sociales como el Facebook, WhatsApp, Instagram, entre otros, y se han convertido en fuente de control en la fase de enamoramiento. 
La pareja, tiene acceso a las claves de las redes sociales, por los celos, fundamentado en el miedo a perder a su enamorada, la mantiene constantemente vigilada a través del seguimiento de sus publicaciones, círculo de amigos, las conversaciones con sus amistades, etc., lo que conlleva a estrangular la relación, y a un control desmedido a través del mundo digital-tecnológico. La manipulan mediante el chantaje emocional, humillándola a través de insultos, llegando hasta el maltrato físico y sexual; intimidándola y amenazándola con hacer público las fotos o videos privados, con lo cual llegan a denigrarla como ser humano.

La manipulación, control y denigración conllevan a un profundo y muy frecuente maltrato psicológico a la mujer en la etapa de enamoramiento. La mujer entra en una fuerte depresión producto de los insultos, humillaciones y baja autoestima, lo que puede conllevar posteriormente al suicidio o feminicidio.

Es importante señalar como este medio tecnológico es un factor desencadenante de violencia de género en parejas jóvenes además de ser el recurso de comunicación más importante para ellos y de comunicación con sus pares.

\section{BIBLIOGRAFÍA}

1. Rugama S, Palacios K, Aragón J. Percepción de los estudiantes de quinto año de Enfermería, acerca de la violencia hacia la mujer [Tesis de licenciatura en internet]. Managua: Universidad Nacional Autónoma de Nicaragua; 2017 [consultado 3 de octubre del 2017]. 132p. Disponible en: http://repositorio.unan.edu.ni/792/1/96652.pdf

2. Aroca C, Ros C, Valera C. Programa para el contexto escolar de prevención de violencia en parejas adolescentes. Educar [Internet]. 2016 [consultado 3 de septiembre
del
2016];
52(1):
$11-31$
Disponible
en: 
http://www.raco.cat/index.php/Educar/article/view/302855/392554

3. Hernández M. Violencia en relaciones de pareja jóvenes [Tesis doctoral en internet]. Valencia: Universidad de Valencia, España; 2012 [consultado el día 10 de septiembre de 2016]. 249p. Disponible en: http://roderic.uv.es/bitstream/handle/10550/24969/tesis\%2012.pdf?sequence=1

4. Martín A, Pazos M, Montilla M, Romero C. Una modalidad actual de violencia de género en parejas de jóvenes: Las redes sociales. Educación XX1[Internet]. 2016. [consultado 3 de septiembre del 2016]; 19(2): 405-29. Disponible en: http://revistas.uned.es/index.php/educacionXX1/article/view/16473/14167

5. Estébanez I. Del amor al control a golpe de click: La violencia de género en las redes sociales. Conferencia presentada en: Jornada de Sensibilización sobre VG del Cabildo de Lanzarote Jornadas "Violencia en género de dudas"; 2012 [Consultado el 15 de diciembre del 2017]; Estella - Lizarra, España. Disponible en: http://minoviomecontrola.com/ianire-estebanez/Ponencia.Del-amor-al-control-a-golpede-click.-La-violencia-de-genero-en-las-redes-sociales.Ianire-Estebanez.pdf

6. Romero C, Montilla C, Martín A. Situaciones vividas sobre violencia de género en adolescentes. Rev. Orientación Educacional [Internet]. 2017 [consultado 8 de Mayo del 2018]; 31(60): 64-84. Disponible en: http://www.roe.cl/index.php/roe/article/view/159/126

7. Blanco M. Implicaciones del uso de las redes sociales en el aumento de la violencia de género en adolescentes. Comunicación y Medios [Internet]. 2015[consultado 8 de Mayo del 2018]; (30): 124-41. Disponible en: https://comunicacionymedios.uchile.cl/index.php/RCM/article/view/32375/37657

8. Grove S, Gray J, Burns N. Investigación en enfermería. Desarrollo de la práctica enfermera basada en la evidencia. 6a ed. Barcelona: Elsevier; 2016. 532p.

9. Do Prado M, De Souza M, Monticelli M, Cometto M, Gómez P. Investigación cualitativa en enfermería. Metodología y didáctica. Washington DC: Organización Panamericana de la Salud; 2013. 253p.

10. INEI (Instituto Nacional de Estadística e Informática). Uso del Internet 
[Internet].Perú. 2017 [Consultado el 4 de Julio de 2018]. Disponible en: https://www.inei.gob.pe/prensa/noticias/el-282-de-la-poblacion-que-usa-internet-lohace-exclusivamente-por-telefono-movil-9825/

11. Donoso T, Rubio M, Vilà R. La Adolescencia ante la violencia de género 2.0: Concepciones, Conductas y Experiencias. Educación XX1 [Internet]. 2017 [consultado 27 de junio de 2018]; 21(1): 109-34. Disponible en: http://revistas.uned.es/index.php/educacionXX1/article/view/20180/16715

12. Rodriguez T, Rodriguez Z. Las nuevas tecnologías: Experiencias de comunicación y conflicto. Nueva época [Internet]. 2016 [Consultado el 2 de diciembre del 2017]; 25 (enero-junio): $15-41$.

Disponible

en: http://www.scielo.org.mx/pdf/comso/n25/n25a2.pdf 